\title{
Measuring the Recoverability of Close Binaries in Gaia DR2 with the Robo-AO Kepler Survey
}

\author{
Carl Ziegler ${ }^{1,2}$ (10), Nicholas M. Law ${ }^{2}$ (1D), Christoph Baranec ${ }^{3}$ (10), Tim Morton ${ }^{4}$, Reed Riddle ${ }^{5}$ (10), Nathan De Lee ${ }^{6,7}$ (1), \\ Daniel Huber ${ }^{3,8,9,10}$, Suvrath Mahadevan ${ }^{11,12}$ (1) , and Joshua Pepper ${ }^{13}$ (1) \\ ${ }^{1}$ Dunlap Institute for Astronomy and Astrophysics, University of Toronto, Ontario M5S 3H4, Canada; carl.ziegler@dunlap.utoronto.ca \\ ${ }^{2}$ Department of Physics and Astronomy, University of North Carolina at Chapel Hill, Chapel Hill, NC 27599-3255, USA \\ ${ }^{3}$ Institute for Astronomy, University of Hawai ‘i at Mānoa, Hilo, HI 96720-2700, USA \\ ${ }^{4}$ Department of Astrophysical Sciences, Princeton University, Princeton, NJ 08544, USA \\ ${ }^{5}$ Division of Physics, Mathematics, and Astronomy, California Institute of Technology, Pasadena, CA 91125, USA \\ ${ }^{6}$ Department of Physics, Geology, and Engineering Technology, Northern Kentucky University, Highland Heights, KY 41099, USA \\ ${ }^{7}$ Department of Physics and Astronomy, Vanderbilt University, Nashville, TN 37235, USA \\ ${ }^{8}$ Sydney Institute for Astronomy (SIfA), School of Physics, University of Sydney, NSW 2006, Australia \\ ${ }^{9}$ SETI Institute, 189 Bernardo Avenue, Mountain View, CA 94043, USA \\ ${ }^{10}$ Stellar Astrophysics Centre, Department of Physics and Astronomy, Aarhus University, Ny Munkegade 120, DK-8000 Aarhus C, Denmark \\ ${ }_{11}$ Department of Astronomy and Astrophysics, The Pennsylvania State University, University Park, PA 16802, USA \\ ${ }^{12}$ Center for Exoplanets and Habitable Worlds, The Pennsylvania State University, University Park, PA 16802, USA \\ ${ }^{13}$ Department of Physics, Lehigh University, 16 Memorial Drive East, Bethlehem, PA 18015, USA \\ Received 2018 June 25; revised 2018 June 29; accepted 2018 July 6; published 2018 November 9
}

\begin{abstract}
We use the Robo-AO survey of Kepler planetary candidate host stars, the largest adaptive optics survey yet performed, to measure the recovery rate of close stellar binaries in Gaia DR2. We find that Gaia recovers binaries down to $1^{\prime \prime}$ at magnitude contrasts as large as six; closer systems are not resolved, regardless of secondary brightness. Gaia DR2 binary detection does not have a strong dependence on the orientation of the stellar pairs. We find 177 nearby stars to Kepler planetary candidate host stars in Gaia DR2 that were not detected in the Robo-AO survey, almost all of which are faint $(G>20)$; the remainder were largely targets observed by Robo-AO in poor conditions. If the primary star is the host, the impact on the radii estimates of planet candidates in these systems is likely minimal; many of these faint stars, however, could be faint eclipsing binaries that are the source of a false positive planetary transit signal. With Robo-AO and Gaia combined, we find that $18.7 \pm 0.7 \%$ of Kepler planet candidate hosts have nearby stars within $4^{\prime \prime}$. We also find 36 nearby stars in Gaia DR2 around 35 planetary candidate host stars detected with $K 2$. The nearby star fraction rate for $K 2$ planetary candidates is significantly lower than that for the primary Kepler mission. The binary recovery rate of Gaia will improve initial radius estimates of future Transiting Exoplanet Survey Satellite planet candidates significantly; however, ground-based high-resolution follow-up observations are still needed for precise characterization and confirmation. The sensitivity of Gaia to closely separated binaries is expected to improve in later data releases.
\end{abstract}

Key words: binaries: close - instrumentation: adaptive optics - methods: data analysis - methods: observational techniques: high angular resolution

Supporting material: machine-readable tables

\section{Introduction}

The Gaia Data Release 2 (DR2) has provided astrometry, parallaxes, and photometry for over a billion stars in the Milky Way (Gaia Collaboration et al. 2018a). Many of these stars are in fact close binaries: approximately half of solar-type stars form with at least one companion (Raghavan et al. 2010; Moe \& Di Stefano 2017). Understanding the multiplicity of stellar populations can provide insight into various stellar formation processes and evolution scenarios (Zhang et al. 2013; Ziegler et al. 2015), as well as provide constraints for theoretical models and massluminosity relationships (Chabrier et al. 2000). The presence of a previously unknown stellar companion to a transiting-planethosting star can substantially increase the estimate of the radius of planets due to the additional flux from the non-transited star (Ciardi et al. 2015; Ziegler et al. 2018a). The Transiting Exoplanet Survey Satellite (TESS, Ricker et al. 2014), with detector pixels $\sim 25 \times$ the size of Kepler, will be particularly susceptible to contamination from nearby sources. In addition, there is significant evidence that stellar binaries can sculpt (Ziegler et al. 2018b) or disrupt (Kraus et al. 2016) planetary systems. Many bound systems have sub-arcsecond separations (Ziegler et al. 2018b) and currently require high-angular resolution instruments on the ground to detect.

With a primary mirror $1.45 \mathrm{~m}$ in size in the scanning direction (Gaia Collaboration et al. 2016), the ability of Gaia to resolve close binaries should be comparable to the Hubble Space Telescope. Gaia Data Release 1 was limited to angular resolutions of $2^{\prime \prime}-4$ " due to data processing limitations (Arenou et al. 2017). DR2 greatly improved on this, sensitive to most $>2^{\prime \prime}$ pairs, but only a small fraction of sub-arcsecond pairs were resolved (Arenou et al. 2018). The probability that Gaia will resolve stellar binaries is not solely a function of separation, however, but also of the flux ratio of the pair and, due to the rectangular pixels of Gaia induced by the scanning direction, the position angle between the two stars (de Bruijne et al. 2015). The close binaries not resolved in DR2 are handled as single objects, with blended photometry and occasional spurious astrometric solutions (Arenou et al. 2018).

There is also the potential for spurious source detections in Gaia DR2. The dominant source of these detections is from 
diffraction spikes around stars brighter than 16 mag (Fabricius et al. 2016). Many of these spurious detections are identified by comparing data from multiple transits (i.e., checking whether the source is consistent in subsequent observations). A fraction of these erroneous detections (less than 20\%) remained in the Gaia Data Release 1, with DR2 expected to be significantly cleaner (Gaia Collaboration et al. 2018a).

The Robo-AO Kepler survey, the largest adaptive optics survey yet performed, with 3857 planetary candidate host stars observed, is an excellent test of the recovery rate of binaries in Gaia DR2. Robo-AO, the first autonomous adaptive optics instrument, detected 620 companions $^{14}$ at separations between 0 ." 15 and 4." 0 and at contrasts up to 7 mag (Law et al. 2014; Baranec et al. 2016; Ziegler et al. 2018a, 2017). The set of Kepler planet candidates host stars are largely $12<G<17$, a brightness regime nearly complete in DR2 (Arenou et al. 2018), and detected companions down to the Gaia faint limit $(G \approx 21)$. With this large homogeneous set of highangular resolution observations, the ability of Gaia to recover binaries as a function of separation, contrast, and orientation can be finely quantified.

We begin in Section 2 by briefly describing the Robo-AO system and the Robo-AO observations of Kepler planetary candidates. We then describe the crossmatching of the RoboAO detections with the Gaia DR2 catalog. We present and discuss the results in Section 3, including the implications for future transiting planet surveys, and conclude in Section 4.

\section{Methodology}

\subsection{Robo-AO Observations}

Observations in the survey were performed using the Robo$\mathrm{AO}$ automated laser adaptive optics system at Palomar and Kitt Peak (Baranec et al. 2014, 2017; Jensen-Clem et al. 2018) that can efficiently perform large, high angular resolution surveys. The adaptive optics system runs at a loop rate of $1.2 \mathrm{kHz}$ to correct high-order wavefront aberrations, delivering median Strehl ratios of $9 \%$ and $4 \%$ in the $i^{\prime}$-band at Palomar and Kitt Peak, respectively. Observations were between 90 and $120 \mathrm{~s}$, and taken in a long-pass filter cutting on at $600 \mathrm{~nm}$. The LP600 filter approximates the Kepler passband at redder wavelengths, while also suppressing blue wavelengths that reduce adaptive optics performance. The LP600 passband is compared to the Kepler passband in Figure 1 of Law et al. (2014). We obtained high-angular-resolution images of 3313 KOIs with Robo-AO between 2012 July 16 and 2015 June 12 (UT) at the Palomar $1.5 \mathrm{~m}$ telescope. We observed 532 additional KOIs with RoboAO between 2016 June 8 and July 15 (UT) at the Kitt Peak $2.1 \mathrm{~m}$ telescope.

\subsection{Gaia-Kepler Crossmatching}

The positions of the Kepler planetary candidates (Mathur et al. 2017; Thompson et al. 2018) were cross-matched on the Gaia online archive service ${ }^{15}$ with an advanced Astronomical Data Query Language search. This provided a list of sources in Gaia DR2 within 5" of each planet candidate host star. To identify the likely primary star in multiple systems, we applied

\footnotetext{
${ }^{14}$ For brevity, we denote stars which we found within our detection radius of KOIs as "companions", in the sense that they are asterisms associated on the sky. For more on the probability of association between each pair of stars, see Ziegler et al. (2018a).

15 http://gea.esac.esa.int/archive/
}

a magnitude cut using the Kepler magnitude of the host star and the Gaia G-magnitude of each source. The star with a $G$-magnitude within 1 mag of the host star's Kepler magnitude was determined to be the primary star. If multiple stars had nearly equivalent brightness, or if no star had a magnitude similar to that in the Kepler catalog, the closest star to the coordinates of the planet candidate host star was determined to be the primary star. In general, the coordinates of the primary star were within 0 " 20 of the positions reported in the Kepler catalog. Several planet candidate host stars had no clear source in Gaia DR2: KOI-98, 227, 640, 959, 1152, and 6728. These systems have been excluded from this analysis.

We searched for potential spurious detections in our crossmatch using the Gaia parallaxes and distance solutions of Bailer-Jones et al. (2018). We found no sources with distances less than $1 \mathrm{pc}$ possibly originating in the solar system or greater than $20 \mathrm{kpc}$ extra-galactic in our sample. Likewise, none had parallaxes greater than $1^{\prime \prime}$ or less than 0.05 mas. None of our sources were found in the catalog of known solar system objects (Gaia Collaboration et al. 2018b). Lastly, the majority of the stars have magnitudes in two additional photometric bands (BP in the blue, and RP in the red) obtained from integrating the Gaia prism spectra. All of the planet candidate hosts and nearby stars with the available photometry had reasonable colors $(-1<(\mathrm{BP}-\mathrm{RP})<4)$, consistent with that of a stellar source (Andrae et al. 2018).

The separation and contrast of any additional sources detected in the area of sky around each host star were compared to the companion properties measured by Robo-AO. The Robo-AO observations were performed between 2012 and 2016 , and the positions of the primary and secondary stars have likely shifted with respect to the Gaia reference epoch (J2015.5). We used the positions and proper motion of the stars detected by Gaia to determine their positions when the Robo-AO observations were performed, using the Astropy software package (The Astropy Collaboration et al. 2018).

The complete list of detections of nearby stars to planet candidate host stars is available in Table 2. Nearby stars in Gaia DR2 with similar contrasts and separations ( $G$-magnitude within 1 magnitude and separations within 0 ".20) to the nearby stars detected using Robo-AO were classified as "recovered", and nearby stars detected with Robo-AO that are not in Gaia DR2 were classified as "not-recovered". We also search for nearby stars in the Gaia DR2 catalog that were not detected by Robo-AO, and list these detections in Table 3 (systems with more than two stars have additional rows for each nearby star). The separation and position angle of these binaries were calculated using the Gaia coordinates using the Astropy software package (The Astropy Collaboration et al. 2018), and the magnitude contrast is calculated from the reported Gaia magnitudes.

In addition to searching around planet candidates from the primary Kepler mission, we also searched Gaia DR2 for sources nearby planetary candidates identified from the ongoing $K 2$ mission. We acquired a list of these planet candidates, 773 in total, and their positions, sourced from EPIC (Huber et al. 2016), from the NASA Exoplanet Archive. ${ }^{16}$ A list of sources from DR2 within 5" of the positions of the candidates was generated, and the host star was identified with magnitude cuts using the

\footnotetext{
16 https://exoplanetarchive.ipac.caltech.edu/
} 
Table 1

Nearby Stars to K2 Planetary Candidates in Gaia DR2

\begin{tabular}{|c|c|c|c|c|c|c|}
\hline $\begin{array}{l}\text { Planet } \\
\text { Candidate }\end{array}$ & $\begin{array}{c}\text { Sep. } \\
\left({ }^{\prime \prime}\right)\end{array}$ & $\begin{array}{l}\text { P.A. } \\
\text { (deg.) }\end{array}$ & $\begin{array}{l}\Delta m_{G} \\
(\mathrm{mag})\end{array}$ & $\begin{array}{c}K 2 \\
\text { Campaign }\end{array}$ & $\begin{array}{l}\text { Primary Gaia } \\
\text { DR2 Source ID }\end{array}$ & $\begin{array}{l}\text { Secondary Gaia } \\
\text { DR2 Source ID }\end{array}$ \\
\hline 202066537.01 & 2.19 & 74 & 0.7 & 0 & 3364627558065966848 & 3364627562364388352 \\
\hline 202086968.01 & 1.95 & 189 & 2.59 & 0 & 3369361402301215616 & 3369361406595494016 \\
\hline 201441872.01 & 3.86 & 240 & 0.37 & 1 & 3797258174978678016 & 3797258174978677888 \\
\hline 201546283.01 & 2.97 & 177 & 5.91 & 1 & 3798552815560689792 & 3798552811267494016 \\
\hline 201637175.01 & 1.92 & 226 & 2.95 & 1 & 3811002791880297600 & 3811002787586327040 \\
\hline 201650711.01 & 1.78 & 332 & 1.35 & 1 & 3812335125095532672 & 3812335125094701056 \\
\hline 201683540.01 & 1.99 & 203 & 3.85 & 1 & 3811900543124260480 & 3811900543123607552 \\
\hline 201828749.01 & 2.45 & 57 & 2.16 & 1 & 3909309851641800704 & 3909309851641320832 \\
\hline 203099398.01 & 1.96 & 64 & 2.49 & 2 & 6042368383828169728 & 6042368388127562752 \\
\hline 205029914.01 & 3.32 & 5 & 1.44 & 2 & 4131047326528868352 & 4131047326531704960 \\
\hline 205029914.01 & 3.72 & 178 & 7.17 & 2 & 4131047326528868352 & 4131047330825537792 \\
\hline 205040048.01 & 3.8 & 330 & 4.25 & 2 & 6245720108744660480 & 6245720104449034880 \\
\hline 205071984.03 & 3.72 & 346 & 6.81 & 2 & 4130539180358512768 & 4130539184653092352 \\
\hline 210625740.01 & 3.6 & 348 & 2.23 & 4 & 46432827015149184 & 46432827013380608 \\
\hline 210666756.01 & 2.4 & 212 & 2.36 & 4 & 49835540624946304 & 49835540624946560 \\
\hline 210958990.01 & 1.81 & 239 & 2.42 & 4 & 52752231438638080 & 52752235733602304 \\
\hline 211509553.01 & 1.96 & 328 & 3.4 & 5 & 605593554127479936 & 605593554127091200 \\
\hline 211694226.01 & 1.81 & 223 & 0.51 & 5 & 609915592602320896 & 609915596898129664 \\
\hline 211791178.01 & 1.67 & 347 & 1.33 & 5 & 659785149366912768 & 659785145072281600 \\
\hline 211978865.01 & 1.08 & 26 & 1.62 & 5 & 675557368789973632 & 675557364493662720 \\
\hline 212398508.01 & 2.32 & 255 & 3.62 & 6 & 3606357633269598464 & 3606357598909037696 \\
\hline 212428509.01 & 1.81 & 73 & 3.33 & 6 & 3606604782867769216 & 3606604782867769344 \\
\hline 212577658.01 & 1.42 & 12 & 0.51 & 6 & 3613738139429952768 & 3613738139430802816 \\
\hline 212628098.01 & 1.85 & 20 & 2.39 & 6 & 3624010185078481664 & 3624010189374241280 \\
\hline 212646483.01 & 1.69 & 213 & 2.66 & 6 & 3615089503644951168 & 3615089507940163072 \\
\hline 212661144.01 & 2.72 & 294 & 2.85 & 6 & 3615758251528391680 & 3615758251528391808 \\
\hline 212679181.01 & 1.24 & 210 & -0.41 & 6 & 3630190784752117504 & 3630190784751508480 \\
\hline 213920015.01 & 1.09 & 198 & -0.09 & 7 & 6764880018721513856 & 6764880018726114688 \\
\hline 214254518.01 & 3.53 & 29 & 7.61 & 7 & 6763711645882517504 & 6763711650189991552 \\
\hline 216114172.01 & 2.62 & 52 & 2.46 & 7 & 6768794138383822848 & 6768794142677222272 \\
\hline 216405287.01 & 2.54 & 254 & 5.59 & 7 & 4078733014274957184 & 4078733014252539008 \\
\hline 217149884.01 & 2.82 & 86 & 5.71 & 7 & 4082665245798510848 & 4082665245790571264 \\
\hline 217855533.01 & 2.49 & 92 & 0.17 & 7 & 4083005128025277952 & 4083005132333049472 \\
\hline 219256848.01 & 2.93 & 253 & 0.0 & 7 & 4088264543134042880 & 4088264543142623104 \\
\hline 219420915.01 & 2.45 & 266 & 4.37 & 7 & 4087971969962403840 & 4087971969954763264 \\
\hline 220192485.01 & 2.3 & 72 & 5.0 & 8 & 2534555412207560960 & 2534555416500291328 \\
\hline
\end{tabular}

(This table is available in machine-readable form.)

Table 2

Robo-AO Detected Nearby Stars to Kepler Planetary Candidates in Gaia DR2

\begin{tabular}{|c|c|c|c|c|c|c|c|}
\hline KOI & $\begin{array}{l}\text { Sep. } \\
\left({ }^{\prime \prime}\right)\end{array}$ & $\begin{array}{l}\text { P.A. } \\
\text { (deg.) }\end{array}$ & $\begin{array}{c}\Delta \mathrm{m} \\
(\mathrm{mags})\end{array}$ & $\begin{array}{l}\text { Detection } \\
\text { reference }\end{array}$ & $\begin{array}{l}\text { Primary Gaia } \\
\text { DR2 source ID }\end{array}$ & $\begin{array}{l}\text { Secondary Gaia } \\
\text { DR2 source ID }\end{array}$ & $\begin{array}{l}\text { Recovered in } \\
\text { Gaia DR2? }\end{array}$ \\
\hline 1 & 1.13 & 135 & 3.95 & L14 & 2131314401800665344 & $\ldots$ & not-recovered \\
\hline 4 & 3.42 & 75 & 4.46 & B16 & 2052194374009877376 & 2052194374009877632 & recovered \\
\hline 13 & 1.16 & 279 & 0.19 & L14 & 2130632159134827392 & 2130632159130638464 & recovered \\
\hline 42 & 1.74 & 35 & 3.04 & B16 & 2106904148451706752 & 2106904148449360000 & recovered \\
\hline 44 & 3.42 & 123 & 4.03 & $\mathrm{Z} 18$ & 2082166236346124544 & 2082166236337520896 & recovered \\
\hline 51 & 3.51 & 161 & 2.63 & $\mathrm{Z} 17$ & 2077023717382584576 & $\cdots$ & not-recovered \\
\hline 70 & 3.86 & 51 & 5.74 & Z18 & 2102548708017562112 & 2102548708017562368 & recovered \\
\hline
\end{tabular}

Note.

Reference for nearby star detection: (L14, Law et al. 2014), (B16, Baranec et al. 2016), (Z17, Ziegler et al. 2017), (Z18, Ziegler et al. 2018a). Provenance of reported companion properties is the detection reference publication.

(This table is available in its entirety in machine-readable form.) 


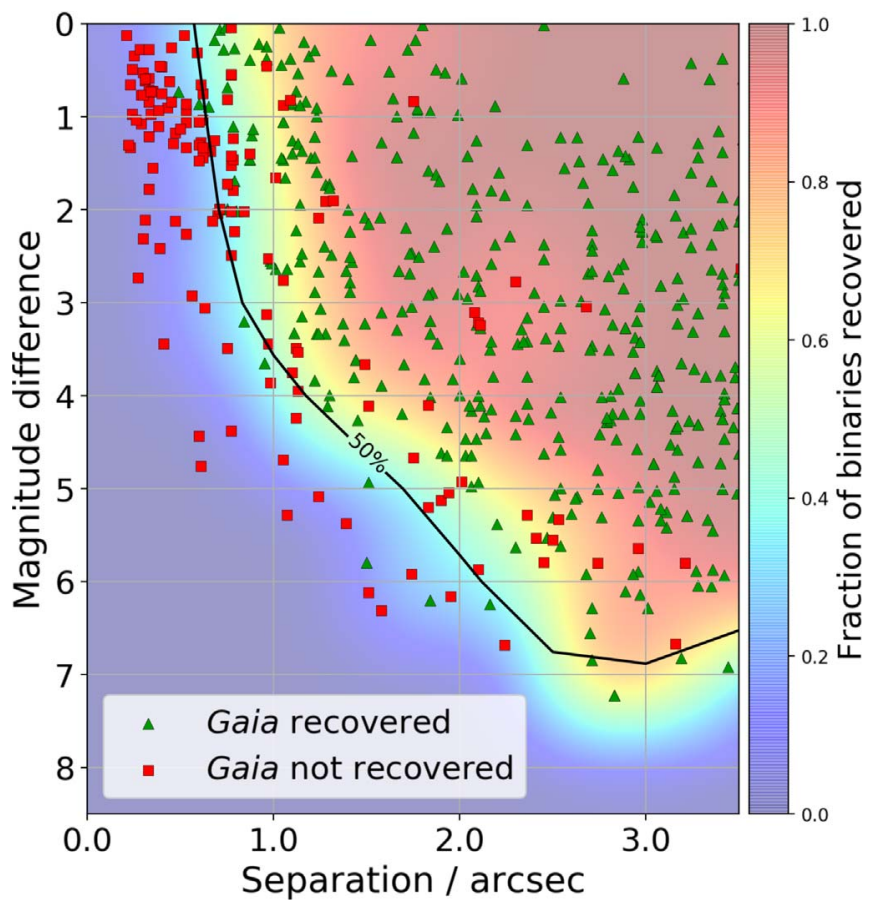

Figure 1. The Robo-AO detections of nearby stars to Kepler planetary candidates recovered and not-recovered in Gaia DR2. The fraction of binaries recovered is plotted as a function of magnitude difference and separation from the primary star, calculated by measuring the number of recovered and nonrecovered stars in bins of size $1 \mathrm{mag}$ and 0.15 and employing a bicubic interpolation. The $50 \%$ recoverability contour has been labeled. In general, stars within $1^{\prime \prime}$ of the primary star are not recovered in Gaia DR2.

Kepler magnitude and Gaia $G$-magnitude. Detections of sources nearby $K 2$ planet candidate host stars are listed in Table 1.

\section{Results and Discussion}

\subsection{Properties of Recovered Stars}

We find that, of the 620 stars detected with Robo-AO within $4^{\prime \prime}$ of 3857 Kepler planetary candidates, 484, or 78\%, appear in Gaia DR2. The recovery classifications for each star is listed in Table 2, along with the Robo-AO measured binary properties and Gaia DR2 source IDs for the primary and recovered secondary stars.

In general, stars within $1^{\prime \prime}$ of the planetary candidate host star were not recovered $(22.4 \%$ recovery rate), and stars at separations greater than $2^{\prime \prime}$ were nearly all recovered $(93 \%$ recovery rate) down to the Gaia faint limit. These recovery rates could potentially be influenced by the ability of Robo-AO to detect binaries at given separations and contrasts in some observations due to low-image performance, resulting from bad seeing or a faint target star. For magnitude contrasts less than three, a region of high completeness for Robo-AO (companions at separations from 0 ! 15 to $4^{\prime \prime}$ are detectable in nearly all images), the recovery rate is $22.9 \%$ within $1^{\prime \prime}$, and $97 \%$ at separations greater than $2^{\prime \prime}$. For Kepler planet candidate hosts, the majority of stars within $1^{\prime \prime}$ are members of likely bound stellar pairs, and their influence can have a significant impact on the architecture of the planetary system (Ziegler et al. 2018b). In Figure 1, we plot the Robo-AO detections recovered and not-recovered by Gaia, as well as the fraction of binaries recovered as a function of magnitude difference and separation.

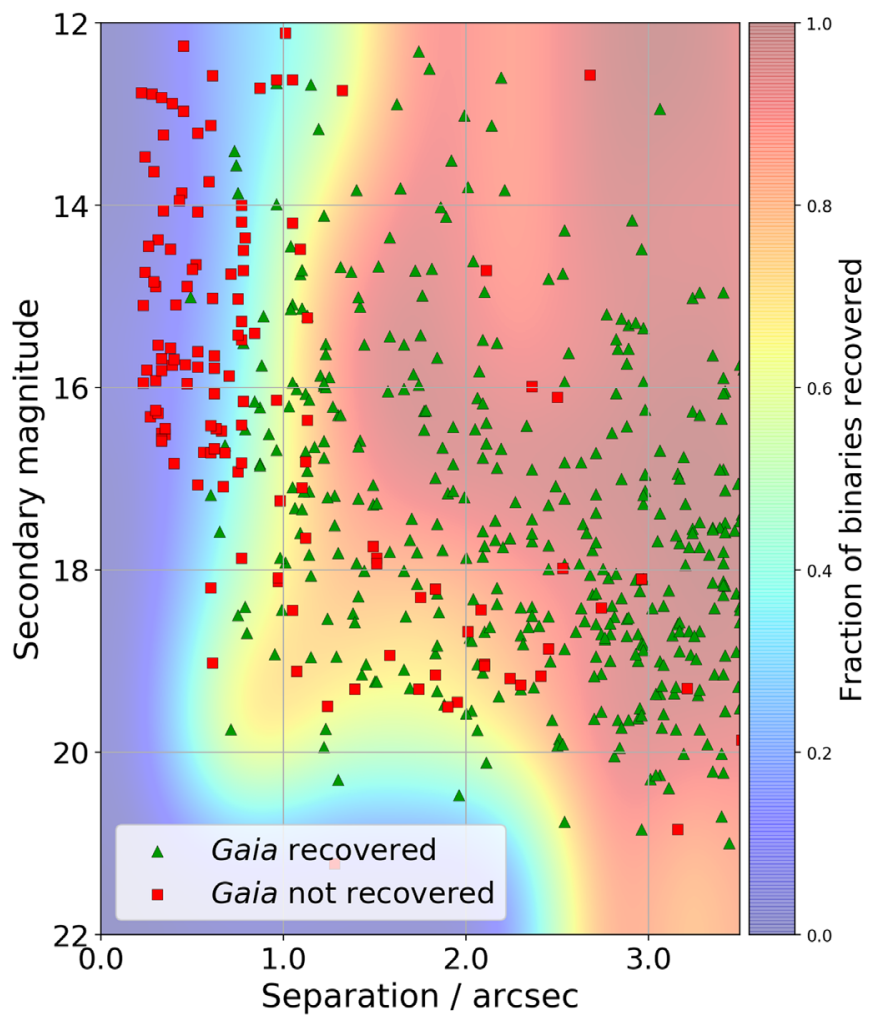

Figure 2. Same as Figure 1, however plotted as a function of the secondary star $G$-magnitude. For recovered binaries, the secondary magnitude was measured by Gaia; for non-recovered binaries, this magnitude is approximate and estimated using the primary star's $G$-magnitude plus the visible contrast measured by Robo-AO. Most stars within $1^{\prime \prime}$ are not recovered, and the recovery rate at low-separations is not dependent on secondary magnitude.

We also find that the recovery rate at low-separations does not depend on the brightness of the secondary star. In Figure 2, we plot the fraction of binaries recovered as a function of the secondary star's magnitude and separation. We find that even at the bright end $\left(m_{G}<13\right)$, very few stars are detected within $1^{\prime \prime}$ of the primary star.

The rectangular Gaia pixels (with a 3:1 size ratio between across-scan and along-scan pixels) may introduce an orientation dependence to the ability of Gaia to resolve close binaries (de Bruijne et al. 2015). This asymmetric sensitivity is not expected to impact the final Gaia catalog, as each object will be observed approximately 70 times at various orientations. However, it may be apparent in the recovery rate of binaries in the DR2 catalog, which is based on 22 months of data collection. In Figure 3, we plot the fraction of stars detected with Robo-AO recovered in Gaia DR2 as a function of position angle. The recovery rates in six position angle bins are all consistent with the overall recovery fraction. If we limit the set to only small-separation binaries $\left(\rho<2^{\prime \prime}\right)$, as most of the variation in recovery will likely occur at these smaller separations, the recovery rate is consistent across all position angles.

\subsection{New Gaia Detections around Kepler Planet Candidates}

Within $4^{\prime \prime}$ of the 3857 Kepler planetary candidate host stars observed by Robo-AO, Gaia DR2 catalogs 177 nearby stars around 163 host stars that were not detected in the Robo-AO survey. The properties of these nearby stars, calculated from the Gaia astrometry and photometry, are listed in Table 3. The 


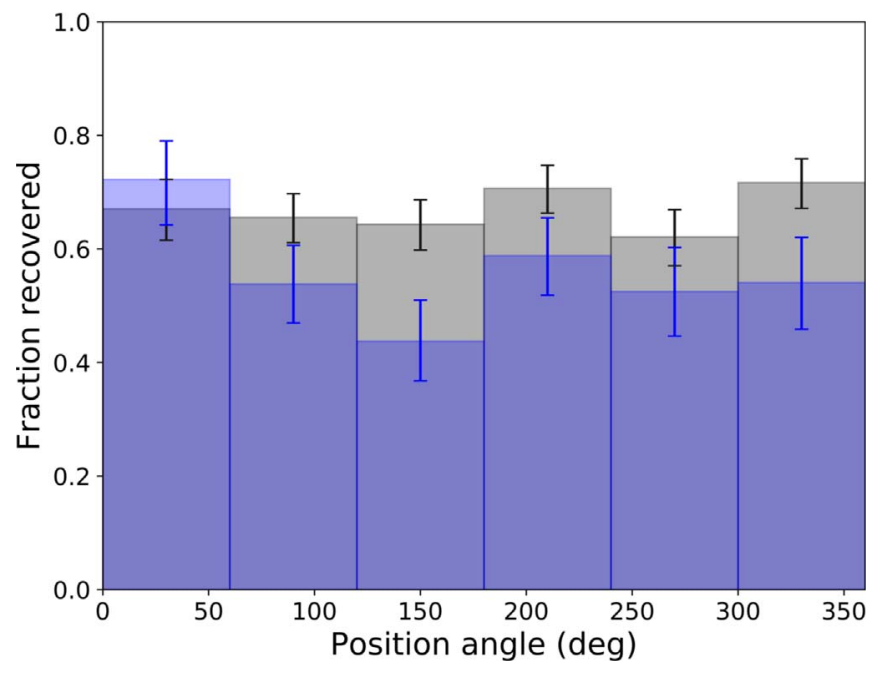

Figure 3. The fraction of nearby stars recovered as a function of position angle with respect to the primary star for all Robo-AO detected stars within $4^{\prime \prime}$ and $2^{\prime \prime}$ in gray and blue, respectively. The recovery rate of nearby stars in Gaia DR2 is not strongly dependent on the position angle of the stars.

majority of these detections fall outside of the sensitivity of Robo-AO, including nearly two-thirds (65\%) fainter than 20 mag. Longer integration times with Robo-AO could potentially observe some of these faint stars. We searched the Robo-AO images for any detection of a companion at the purported position of the nearby stars detected by Gaia (accounting for proper motion shift). None were detected with $5 \sigma$ significance; however, several low-significance detections were apparent to visual inspection. A future study using highresolution data from a large-aperture telescope (such as Keck$\mathrm{AO}$ ) could potentially determine the validity of these faint Gaia detections nearby bright stars.

Altogether, approximately $99.5 \%$ of secondary stars with $G<18$ detected by Gaia were also detected in the Robo-AO Kepler survey. The exceptions, all from particularly lowperformance observations with shallow contrast curves, are secondaries in DR2 nearby KOI-118, 433, and 5736. The properties of the new detections are plotted in Figure 4, along with typical Robo-AO visible-light contrast curves for three image performance groups, determined using the PSF core size as described in Law et al. (2014).

The Robo-AO Kepler survey found a nearby star fraction rate of $14.5 \pm 0.6 \%$ in the Robo-AO detectability range (separations between $\sim 0$ !" 15 and 4 !' 0 and $\Delta m \leqslant 6$ ). With the additional nearby stars in Gaia DR2 combined with the Robo-AO detections, the nearby star rate of Kepler planet candidate hosts is $18.7 \pm 0.7 \%$. Outside of $1^{\prime \prime}$, where Gaia recovers the majority of binaries, the nearby star fraction rate for Robo-AO and Gaia is $11.3 \pm 0.5 \%$.

\subsection{Kepler Planetary Candidate Radii}

A nearby star in the same photometric aperture as the target star will dilute the observed transit depth, resulting in underestimated radius estimates. In systems with a detected nearby star by Robo-AO, the estimated planetary radius will increase by a factor of 2.18 , on average, if either star is assumed to be equally likely to host the planet Ziegler et al. (2018a). For just systems with likely bound stars, determined with photometric parallaxes, the radii will increase by a factor of 1.77, on average (Ziegler et al. 2018b).

The nearby stars in Gaia DR2 that were not detected by Robo-AO are, in general, faint and widely separated from the host star. Galactic simulations suggest that the majority of these stars are likely not bound to the primary star (Horch et al. 2014; Ziegler et al. 2018b). Assuming the planet indeed orbits the primary star, we use the relation from Law et al. (2014) to correct for the transit dilution,

$$
R_{p, A}=R_{p, 0} \sqrt{\frac{1}{F_{A}}}
$$

where $R_{p, A}$ is the corrected radius of the planet orbiting the primary star, $R_{p, 0}$ is the original planetary radius estimate based on the diluted transit signal, and $F_{A}$ is the fraction of flux within the aperture from the primary star.

With the high contrasts of the newly detected Gaia stars, their contamination of the Kepler light curves is minimal. Using the Gaia photometry as a proxy for the Kepler photometry, if the transiting planet candidates orbit the primary star, their radii will increase by a factor of 1.007 due to the additional flux from these faint stars.

If instead, these planets orbit the secondary star, the corrected planet radius estimate relies on the radius of the secondary star, which is generally not known without color information. If we assume that all nearby stars are bound to the primary star, and use as the secondary radius the radius of an appropriately fainter star within the Dartmouth stellar models (Dotter et al. 2008), we can use the relation

$$
R_{p, B}=R_{p, 0} \frac{R_{B}}{R_{A}} \sqrt{\frac{1}{F_{B}}}
$$

where $R_{p, B}$ is the corrected radius of the planet orbiting the secondary star bound to the primary star, $R_{B}$ and $R_{A}$ are the stellar radii of the secondary and primary star, respectively, and $F_{B}$ is the fraction of flux within the aperture from the secondary star. In this scenario, the planetary radii will increase by, on average, a factor of 8.2 in these systems. This scenario is unrealistic, however, and leads to a planetary population with a large fraction of gas giants, which is inconsistent with the understood planet occurrence rates of the galaxy (Howard et al. 2012). This scenario should be investigated for rare, difficult-to-model systems, such as those with unlikely dynamical properties, where one or more planet candidates could, in fact, be associated with the secondary star.

\subsection{Nearby Stars in Gaia to K2 Planet Candidates}

We searched for nearby stars in the Gaia DR2 catalog around $773 K 2$ planet candidates from the first eight $K 2$ campaigns, as listed on the NASA Exoplanet Archive. We found 36 nearby stars around 35 planet candidate hosts. The properties of these detected stars are listed in Table 1 and plotted in Figure 5.

The fraction of nearby stars in Gaia DR2 to $K 2$ planetary candidates $(4.5 \%)$ is significantly lower than that of Kepler planet candidates $(9.7 \%)$. The disparity between the nearby star fraction of Kepler and $K 2$ planet candidate hosts may be due to the $K 2$ fields, which follow the ecliptic, appearing in less dense stellar regions with fewer unassociated background or foreground stars. The $K 2$ targets lie, on average, at approximately 
Table 3

New Nearby Stars to Kepler Planetary Candidates in Gaia DR2

\begin{tabular}{|c|c|c|c|c|c|}
\hline KOI & $\begin{array}{c}\text { Sep. } \\
\left({ }^{\prime \prime}\right)\end{array}$ & $\begin{array}{l}\text { P.A. } \\
\text { (deg.) }\end{array}$ & $\begin{array}{l}\Delta m_{G} \\
(\mathrm{mag})\end{array}$ & $\begin{array}{l}\text { Primary Gaia } \\
\text { DR2 Source ID }\end{array}$ & $\begin{array}{l}\text { Secondary Gaia } \\
\text { DR2 Source ID }\end{array}$ \\
\hline 18 & 3.48 & 111 & 7.27 & 2079018300195390464 & 2076015877539239680 \\
\hline 118 & 1.46 & 212 & 4.34 & 2099605968225288960 & 2099605968225289088 \\
\hline 217 & 3.9 & 326 & 5.16 & 2080095679848047872 & 2080095684146710400 \\
\hline 221 & 1.57 & 276 & 5.28 & 2100073393808483328 & 2100073398103782272 \\
\hline 235 & 3.54 & 13 & 5.64 & 2078125359313871488 & 2078125359309472768 \\
\hline 266 & 3.74 & 325 & 8.16 & 2078008703696509056 & 2078008707991780352 \\
\hline 344 & 3.57 & 210 & 6.87 & 2131736137528127744 & 2131736137526502656 \\
\hline 354 & 3.77 & 209 & 6.59 & 2134886100904948608 & 21348860666539848832 \\
\hline 415 & 3.36 & 208 & 6.63 & 2077596288060821120 & 2077596288054326656 \\
\hline 433 & 2.38 & 5 & 4.02 & 2086498312157538688 & 2086498312152241280 \\
\hline 433 & 3.72 & 291 & 2.65 & 2086498312157538688 & 2086498312152239104 \\
\hline 488 & 3.2 & 260 & 6.04 & 2099156817725596800 & 2099156817721708416 \\
\hline 497 & 1.96 & 306 & 5.33 & 2076454926274192128 & 2076454926274445312 \\
\hline 500 & 1.69 & 158 & 5.26 & 2076328963475704576 & 2076328963464397696 \\
\hline 533 & 2.82 & 255 & 5.82 & 2119809116425132160 & 2119809116422789504 \\
\hline 624 & 3.79 & 38 & 6.13 & 2052852912747829760 & 2052852912747830912 \\
\hline 683 & 3.35 & 268 & 6.07 & 2078640132611129088 & 2078640136909689216 \\
\hline 689 & 3.89 & 212 & 6.58 & 2126970579257130112 & 2126970579251947904 \\
\hline 753 & 3.71 & 200 & 5.4 & 2086623622121548800 & 2086623622116676096 \\
\hline 767 & 2.77 & 92 & 4.74 & 2086830502105850240 & 2086830605181608704 \\
\hline 893 & 2.27 & 276 & 5.23 & 2125888419299436800 & 2125888419294076032 \\
\hline 908 & 1.46 & 197 & 4.35 & 2079056714383865856 & 2079056714376186752 \\
\hline 1031 & 3.5 & 109 & 5.56 & 2051669906960994304 & 2051669902665001344 \\
\hline 1094 & 3.49 & 208 & 4.57 & 2052234368740841600 & 2052234368730056448 \\
\hline 1099 & 3.02 & 234 & 5.3 & 2052567623840745216 & 2052567623838348672 \\
\hline 1101 & 1.7 & 338 & 3.72 & 2052074389802779904 & 2052074389798914688 \\
\hline 1102 & 2.91 & 141 & 4.63 & 2052823535171095296 & 2052823530876477824 \\
\hline 1146 & 3.87 & 35 & 4.99 & 2105915343901864832 & 2105915343899024512 \\
\hline 1165 & 3.81 & 309 & 5.99 & 2129164173675031936 & 2129164173672715904 \\
\hline 1199 & 3.2 & 92 & 5.7 & 2052392835854583936 & 2052392835843568896 \\
\hline 1210 & 3.91 & 13 & 5.66 & 2052397886736075264 & 20523978866724924800 \\
\hline 1212 & 3.72 & 251 & 4.76 & 2052717466660745984 & 20527174666654426240 \\
\hline 1216 & 3.11 & 77 & 6.5 & 2099681216051775616 & 2099681216046506368 \\
\hline 1230 & 2.77 & 108 & 6.31 & 2075373036891027968 & 2075373041187294080 \\
\hline 1242 & 3.75 & 169 & 6.12 & 2125716650665286144 & 2125716654965607680 \\
\hline 1245 & 3.67 & 101 & 5.81 & 2125709405062046976 & 2125709405055099264 \\
\hline 1257 & 3.1 & 4 & 5.67 & 2126633166627436928 & 2126633166623505152 \\
\hline 1321 & 3.06 & 101 & 4.96 & 2076223479079929984 & 2076223479070585216 \\
\hline 1323 & 2.52 & 60 & 3.82 & 2073195870740245504 & 2073195870733315456 \\
\hline 1325 & 3.53 & 17 & 4.47 & 2073292868278609664 & 2073292868269699456 \\
\hline 1339 & 2.66 & 311 & 5.95 & 2100216609495590912 & 2100216609493017216 \\
\hline 1408 & 3.5 & 3 & 6.02 & 2127463224886292224 & 2127463229186009088 \\
\hline 1428 & 2.63 & 170 & 5.38 & 2129939398090453504 & 2129939398091259520 \\
\hline 1448 & 3.72 & 293 & 4.92 & 2127712474727909504 & 2127712474723372928 \\
\hline 1455 & 3.29 & 244 & 4.81 & 2076280447527786880 & 2076280447522669696 \\
\hline 1472 & 3.35 & 358 & 4.41 & 2078176589682140160 & 2078176589677446272 \\
\hline 1499 & 2.97 & 257 & 5.12 & 2078648211455026432 & 2078648177089867520 \\
\hline 1517 & 3.71 & 130 & 5.22 & 2077967888633787264 & 20779678886633787392 \\
\hline 1552 & 3.24 & 226 & 4.15 & 2078982634786330112 & 2078982531697453056 \\
\hline 1581 & 3.07 & 28 & 4.83 & 2105221139747765504 & 2105221139745555968 \\
\hline 1615 & 2.96 & 356 & 7.24 & 2076194101502797952 & 2076194101502799104 \\
\hline 1637 & 1.14 & 340 & 3.75 & 2085724496490595584 & 2085724496486440576 \\
\hline 1664 & 3.58 & 304 & 4.84 & 2053298489838427264 & 2053298489831008768 \\
\hline 1761 & 2.45 & 307 & 4.99 & 2052387815030027904 & 2052387819323056128 \\
\hline 1762 & 3.36 & 187 & 5.03 & 2073774381339451904 & 2073774385635104128 \\
\hline 1762 & 3.86 & 352 & 5.04 & 2073774381339451904 & 2073774385649871872 \\
\hline 1793 & 2.97 & 55 & 4.43 & 2073821767730332928 & 2073823245188434688 \\
\hline 1821 & 3.82 & 90 & 5.84 & 2079728790863754752 & 2079728790858521600 \\
\hline 1852 & 2.6 & 259 & 6.24 & 2130393938771375104 & 2130393943069501312 \\
\hline 1910 & 2.09 & 174 & 5.5 & 2102899314789524480 & 2102899314789616768 \\
\hline 1935 & 1.75 & 151 & 3.56 & 2075043909268197120 & 2075043909249776128 \\
\hline 1967 & 3.02 & 152 & 5.33 & 2101670026430316416 & 2101670026430316160 \\
\hline 1997 & 2.36 & 168 & 5.58 & 2119617320366308736 & 2119617320366308608 \\
\hline
\end{tabular}


Table 3

(Continued)

\begin{tabular}{|c|c|c|c|c|c|}
\hline KOI & $\begin{array}{c}\text { Sep. } \\
\left({ }^{\prime \prime}\right)\end{array}$ & $\begin{array}{l}\text { P.A. } \\
\text { (deg.) }\end{array}$ & $\begin{array}{l}\Delta m_{G} \\
(\mathrm{mag})\end{array}$ & $\begin{array}{l}\text { Primary Gaia } \\
\text { DR2 Source ID }\end{array}$ & $\begin{array}{l}\text { Secondary Gaia } \\
\text { DR2 Source ID }\end{array}$ \\
\hline 2130 & 2.1 & 22 & 4.12 & 2051797274209237376 & 2051797278505487616 \\
\hline 2130 & 1.8 & 232 & 3.92 & 2051797274209237376 & 2051797278505484928 \\
\hline 2137 & 3.12 & 2 & 6.57 & 2085258750237006208 & 2085258750234401536 \\
\hline 2146 & 2.34 & 235 & 5.53 & 2101941434002832000 & 2101941433996820480 \\
\hline 2199 & 2.3 & 53 & 4.62 & 2132842177507335424 & 2132842177503715712 \\
\hline 2210 & 3.29 & 90 & 5.11 & 2100405381897402496 & 2100405381894221184 \\
\hline 2241 & 3.77 & 331 & 4.6 & 2073767994738269952 & 2073767994722471808 \\
\hline 2259 & 3.83 & 357 & 5.42 & 2076050683953049728 & 2076050683942049792 \\
\hline 2328 & 3.24 & 349 & 4.66 & 2105273705848965888 & 2105273710146215424 \\
\hline 2344 & 2.7 & 120 & 4.57 & 2078832929407597312 & 2078832929407597952 \\
\hline 2373 & 2.47 & 104 & 5.97 & 2129265088226500224 & 2129265088224647552 \\
\hline 2494 & 1.81 & 131 & 5.24 & 2077894083914484352 & 2077894083909574400 \\
\hline 2507 & 2.26 & 275 & 3.79 & 2079680137473255808 & 2079680137465012224 \\
\hline 2519 & 2.88 & 345 & 5.99 & 2099514502602729600 & 2099514502599632512 \\
\hline 2553 & 3.16 & 189 & 5.14 & 2128318305636573824 & 2128318305632504832 \\
\hline 2617 & 3.22 & 151 & 5.01 & 2101249188353780480 & 2101249188348398464 \\
\hline 2643 & 3.27 & 124 & 4.58 & 2105463135385330944 & 2105463135381131648 \\
\hline 2734 & 2.35 & 89 & 3.39 & 2116889775616597120 & 2116886820677495552 \\
\hline 2822 & 2.26 & 34 & 4.24 & 2076067073548982784 & 2076067073540267136 \\
\hline 2865 & 3.21 & 66 & 4.85 & 2127136605512921600 & 2127136983466106112 \\
\hline 2942 & 3.05 & 148 & 3.43 & 2073294414466892288 & 2073294414460099584 \\
\hline 2982 & 3.01 & 17 & 6.16 & 2126892067256468736 & 2126892067252859008 \\
\hline 3048 & 3.52 & 152 & 5.03 & 2128168256659148032 & 2128168256652877312 \\
\hline 3050 & 3.88 & 177 & 5.45 & 2073778646257819520 & 2073778646244536576 \\
\hline 3065 & 2.35 & 217 & 5.59 & 2073569356783775488 & 2073569361099660416 \\
\hline 3065 & 3.93 & 75 & 5.74 & 2073569356783775488 & 2073569361088192128 \\
\hline 3117 & 2.72 & 285 & 5.86 & 2101695418277116672 & 2101695418275679488 \\
\hline 3119 & 1.12 & 313 & 2.91 & 2076747533796560256 & 2076747533807064448 \\
\hline 3128 & 3.76 & 191 & 6.54 & 2125872270222128512 & 2125872270216008448 \\
\hline 3259 & 2.17 & 326 & 4.79 & 2052579546669972096 & 20525795466666328704 \\
\hline 3271 & 3.73 & 4 & 4.64 & 2125848733801094912 & 2125848733801095296 \\
\hline 3346 & 2.79 & 0 & 6.21 & 2129558760909448064 & 2129558760906481536 \\
\hline 3482 & 3.53 & 297 & 5.0 & 2077774443305045376 & 2077774443301405312 \\
\hline 3692 & 2.27 & 153 & 4.75 & 2073819156390137088 & 2073819156379156736 \\
\hline 3692 & 3.03 & 264 & 4.82 & 2073819156390137088 & 2073819156379156992 \\
\hline 3709 & 2.26 & 93 & 3.94 & 2052564394025218432 & 2052564325305742080 \\
\hline 3818 & 3.73 & 76 & 7.0 & 2102620829109177856 & 2102620829103353088 \\
\hline 3939 & 1.93 & 165 & 4.96 & 2128115754978992640 & 2128115754978992512 \\
\hline 4002 & 3.65 & 216 & 5.23 & 2103829913941569664 & 2103829913937915136 \\
\hline 4016 & 3.34 & 186 & 5.71 & 2104452752917443584 & 2104452748621944960 \\
\hline 4056 & 2.58 & 32 & 5.07 & 2073307917844452480 & 2073307917836893184 \\
\hline 4120 & 3.44 & 135 & 5.57 & 2130342609620849024 & 2130342609620849280 \\
\hline 4121 & 2.12 & 86 & 4.45 & 2103927495598786304 & 2103927495595046656 \\
\hline 4136 & 3.68 & 108 & 6.21 & 2104828820254417664 & 2104828820251807232 \\
\hline 4156 & 3.81 & 315 & 5.9 & 2077381299180152704 & 2077381299174112256 \\
\hline 4173 & 3.36 & 57 & 5.56 & 2085325373768675968 & 2085325373759050496 \\
\hline 4288 & 2.94 & 279 & 7.17 & 2100418850915010432 & 2100418850912250752 \\
\hline 4302 & 3.39 & 118 & 6.46 & 2077605049794388736 & 2077605049788794240 \\
\hline 4307 & 3.22 & 194 & 6.36 & 2052136821446989568 & 2052136821437832320 \\
\hline 4393 & 1.44 & 84 & 3.63 & 2079025996777083776 & 2079025996772785920 \\
\hline 4452 & 3.17 & 85 & 5.93 & 2052436919400076672 & 2052436919392240128 \\
\hline 4458 & 1.5 & 60 & 4.14 & 2076141428024552192 & 2076141428021401088 \\
\hline 4473 & 3.43 & 198 & 6.18 & 2077241558125147904 & 2077241558116766464 \\
\hline 4504 & 1.35 & 44 & 4.1 & 2101728197467726848 & 2101728197462775296 \\
\hline 4545 & 2.21 & 137 & 5.26 & 2129106724192000512 & 2129106724188370048 \\
\hline 4591 & 2.76 & 184 & 4.91 & 2073589461547090048 & 2073589461547089408 \\
\hline 4605 & 3.58 & 37 & 6.07 & 2133210307743921024 & 2133210307741784064 \\
\hline 4659 & 2.62 & 102 & 6.57 & 2051918293507634048 & 2051918293508096384 \\
\hline 4754 & 3.71 & 261 & 4.95 & 2086348641136640512 & 2086348641131299072 \\
\hline 4782 & 1.76 & 51 & 3.5 & 2079323723908273408 & 2079323723903496960 \\
\hline 4799 & 3.51 & 283 & 5.98 & 2117304222780856832 & 2117304188417929856 \\
\hline 4875 & 1.78 & 20 & 3.12 & 2052619541406162304 & 2052619541402269696 \\
\hline 4886 & 2.86 & 318 & 4.87 & 2078077977233913344 & 2078077977226315136 \\
\hline
\end{tabular}


Table 3

(Continued)

\begin{tabular}{|c|c|c|c|c|c|}
\hline KOI & $\begin{array}{c}\text { Sep. } \\
\left({ }^{\prime \prime}\right)\end{array}$ & $\begin{array}{l}\text { P.A. } \\
\text { (deg.) }\end{array}$ & $\begin{array}{l}\Delta m_{G} \\
(\mathrm{mag})\end{array}$ & $\begin{array}{l}\text { Primary Gaia } \\
\text { DR2 Source ID }\end{array}$ & $\begin{array}{l}\text { Secondary Gaia } \\
\text { DR2 Source ID }\end{array}$ \\
\hline 4887 & 2.67 & 124 & 6.26 & 2053103837616774528 & 2053103841919097728 \\
\hline 4962 & 3.31 & 198 & 6.36 & 2052111395239338624 & 2052111395226557184 \\
\hline 5031 & 3.03 & 316 & 6.67 & 2076172008190962432 & 2076172008179490048 \\
\hline 5033 & 3.06 & 86 & 5.62 & 2073274314019537152 & 2073274314020402176 \\
\hline 5067 & 3.16 & 305 & 4.28 & 2076246190868032000 & 2076246190861696128 \\
\hline 5085 & 3.48 & 357 & 4.2 & 2076276633596891648 & 2076276633591957888 \\
\hline 5107 & 3.12 & 206 & 6.06 & 2076333567680646400 & 2076333567669338240 \\
\hline 5123 & 2.74 & 185 & 6.19 & 2101258259324916096 & 2101258259319751936 \\
\hline 5158 & 2.64 & 15 & 6.63 & 2075052048214199168 & 2075052052526639744 \\
\hline 5279 & 2.33 & 175 & 5.73 & 2073906705005532672 & 2073906705005532416 \\
\hline 5279 & 1.26 & 64 & 3.9 & 2073906705005532672 & 2073906705001120896 \\
\hline 5279 & 3.42 & 39 & 5.52 & 2073906705005532672 & 2073906705001126656 \\
\hline 5308 & 3.6 & 56 & 6.09 & 2075427089068650368 & 2075427123428777216 \\
\hline 5317 & 2.99 & 132 & 6.2 & 2077530867121103232 & 2077530867118047744 \\
\hline 5384 & 3.03 & 353 & 4.15 & 2078000702181387392 & 2078000702179708544 \\
\hline 5508 & 1.38 & 178 & 4.66 & 2126188036217701504 & 2126188036214524672 \\
\hline 5574 & 1.82 & 219 & 4.76 & 2127024180449752064 & 2127024180446115456 \\
\hline 5574 & 2.88 & 297 & 4.41 & 2127024180449752064 & 2127024180446115712 \\
\hline 5628 & 3.6 & 350 & 5.24 & 2126534313661335040 & 2126534313658794112 \\
\hline 5718 & 3.85 & 250 & 2.87 & 2080475840294368896 & 2080475840290135424 \\
\hline 5732 & 3.71 & 301 & 5.16 & 2085558882551597184 & 2085558882544801152 \\
\hline 5733 & 3.26 & 113 & 4.72 & 2085515245682829440 & 2085515245682829824 \\
\hline 5736 & 0.84 & 304 & 2.22 & 2107550317687202048 & 2107550317690423808 \\
\hline 5796 & 3.01 & 140 & 5.4 & 2129230762847028096 & 2129230762842177408 \\
\hline 5825 & 3.5 & 336 & 6.47 & 2128744057154644736 & 2128744057150059520 \\
\hline 5835 & 1.87 & 135 & 4.88 & 2129409948882314880 & 2129409948878328960 \\
\hline 6102 & 3.22 & 46 & 7.57 & 2052738288662716928 & 2052738288654778752 \\
\hline 6266 & 2.36 & 242 & 5.14 & 2051824869377151104 & 2051824869377150976 \\
\hline 6266 & 3.74 & 112 & 5.4 & 2051824869377151104 & 2051824899439939968 \\
\hline 6385 & 2.23 & 283 & 4.74 & 2073185150501623296 & 2073185150495364480 \\
\hline 6399 & 2.88 & 179 & 4.63 & 2052420083127990272 & 2052420083127990016 \\
\hline 6455 & 2.65 & 200 & 6.13 & 2101105599008028160 & 2101105599008615040 \\
\hline 6484 & 3.55 & 19 & 4.99 & 2100690464646750848 & 2100690464643697536 \\
\hline 6484 & 2.63 & 204 & 4.93 & 2100690464646750848 & 2100690464645431680 \\
\hline 6542 & 2.53 & 40 & 7.14 & 2073583104995090304 & 2073583104995524096 \\
\hline 6542 & 3.03 & 199 & 7.18 & 2073583104995090304 & 2073583104995391488 \\
\hline 6707 & 3.91 & 6 & 3.78 & 2101867594925534208 & 2101867629280609280 \\
\hline 6904 & 1.52 & 277 & 2.56 & 2078219642433457152 & 2078219642426179840 \\
\hline 6944 & 2.98 & 210 & 6.14 & 2078058941938623616 & 2078058941936124672 \\
\hline 7051 & 3.4 & 112 & 4.5 & 2081916578488487808 & 2081916509765722112 \\
\hline 7197 & 2.69 & 109 & 4.61 & 2080091664059732480 & 2080091664056155520 \\
\hline 7223 & 3.99 & 329 & 4.87 & 2085335853489145728 & 2085335853483598592 \\
\hline 7223 & 3.8 & 93 & 5.69 & 2085335853489145728 & 2085335853489146624 \\
\hline 7272 & 2.66 & 213 & 5.33 & 2080510268752316672 & 2080510268748439936 \\
\hline 7445 & 2.75 & 283 & 4.97 & 2134865347623098368 & 2134865347618020736 \\
\hline 7596 & 3.08 & 278 & 4.3 & 2127160000197425024 & 2127160004490984064 \\
\hline
\end{tabular}

Note. Provenance of reported companion properties is from Gaia DR2 (Gaia Collaboration et al. 2018a).

(This table is available in machine-readable form.)

$|b|=38^{\circ}$, far from the galactic plane and a significantly less dense region of the sky than the primary Kepler mission (which had a center of field at $b=14^{\circ}$ ). In addition, Gaia operates with a scanning law that passes through the north and south ecliptic poles every six hours, resulting in over twice as many observations at high ecliptic latitudes, such as the original Kepler field, as at the ecliptic plane, where the $K 2$ fields lie (Gaia Collaboration et al. 2016). The additional observations likely improved the sensitivity of Gaia to closely separated stars in the $K 2$ fields (de Bruijne et al. 2015).

Lastly, part of the disparity between the two samples may also in part be due to the larger fraction of late-type stars in $K 2$
(Huber et al. 2016), which have, at these distances, an adaptive optics resolvable binarity rate of approximately half that of solar-type stars (Law et al. 2005; Janson et al. 2012). Indeed, only 2 of the $36(5.5 \%)$ nearby stars to $K 2$ candidates in Gaia DR2 lie at separations less than 1."5, compared to 78 of 420 $(17.3 \%)$ for the Kepler candidates, consistent with a low inherent binarity rate in the $K 2$ sample.

Crossfield et al. (2016) observed in high-resolution 164 of the candidate planets from $K 2$ campaigns $0-4$ using Keck-AO, Palomar PHARO/PALM-3000, LBT-LMIRCam, Gemini-NIRI, and Robo-AO. Within the separation range in which Gaia has high binary recovery rate $\left(1^{\prime \prime}-4^{\prime \prime}\right), 22$ nearby stars were detected 


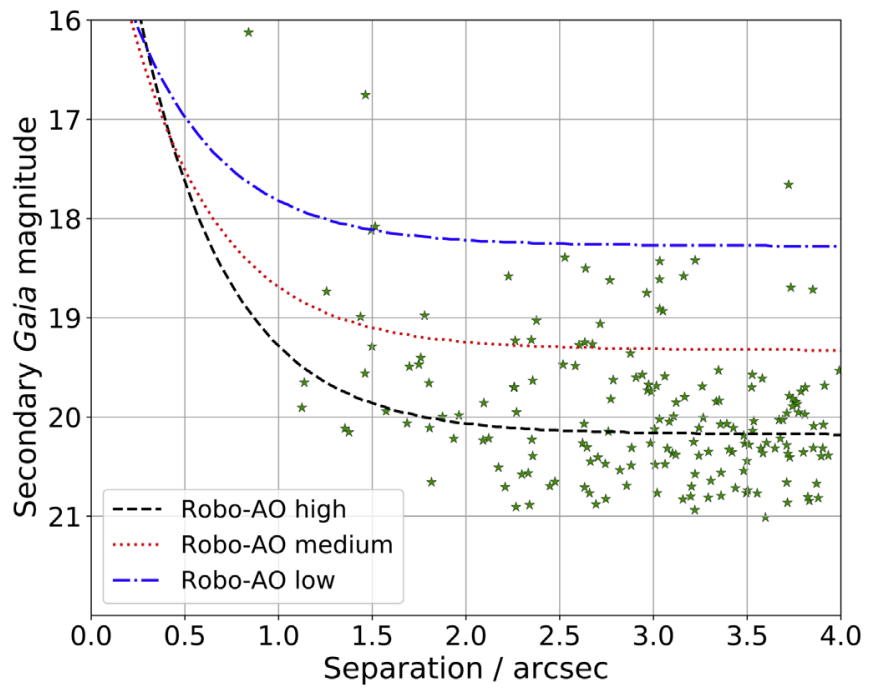

Figure 4. Nearby stars to Kepler planetary candidates in Gaia DR2 that were not detected in Robo-AO images. Typical contrast curves for Robo-AO, in approximate Gaia $g$-band magnitudes, are included for three image performance groups. The majority of these nearby stars were too faint for a significant detection in the Robo-AO images.

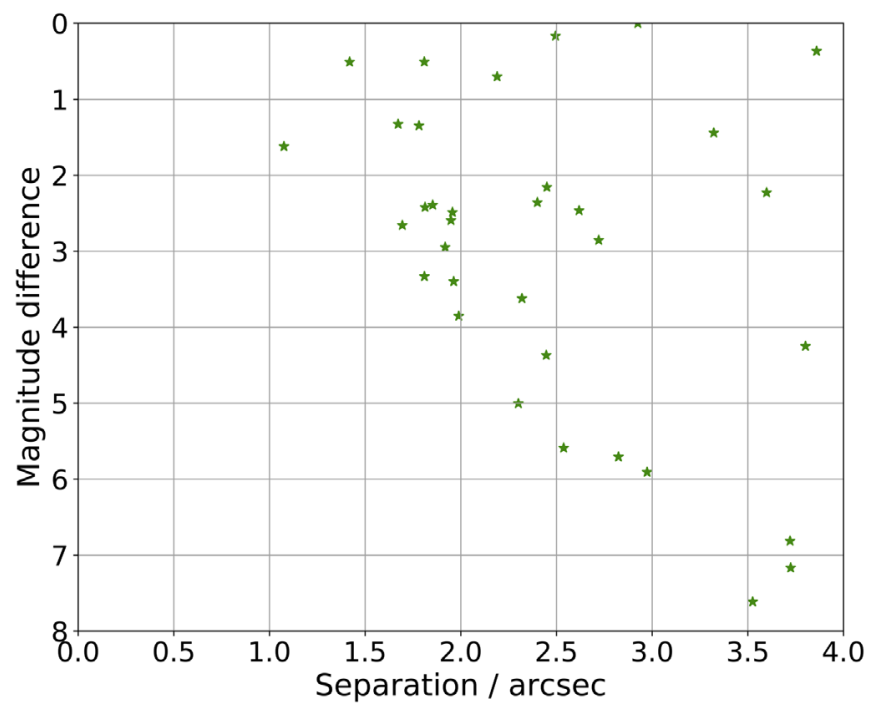

Figure 5. Nearby stars to $K 2$ planetary candidates in Gaia DR2. The nearby star rate of $K 2$ planet candidates is less than half that of Kepler planet candidates in Gaia DR2.

around 20 planet candidate hosts, for a nearby star fraction rate of $12.2 \%$. Only 7 of the 20 multiple systems were detected by Gaia: EPIC 201546283, 201828749, 202066537, 205029914, $210666756,210958990,203099398$. This recovery rate $(35 \%)$ is significantly less than that for Kepler planet candidates within the same separation range $(82 \%)$.

The reason for the low binary recovery rate of Gaia DR2 compared to the high-resolution imaging in the $K 2$ fields is unclear. Arenou et al. (2018) found that DR2 recovered significantly more close binaries in low-density fields, similar to the first five $K 2$ campaign fields. The majority of the observations performed in Crossfield et al. (2016) were done in the NIR, with 10 of 13 of the binary systems not detected in Gaia DR2 having contrasts greater than a magnitude of five. It is possible that the secondary stars in these systems are below the Gaia faint limit in the visible.

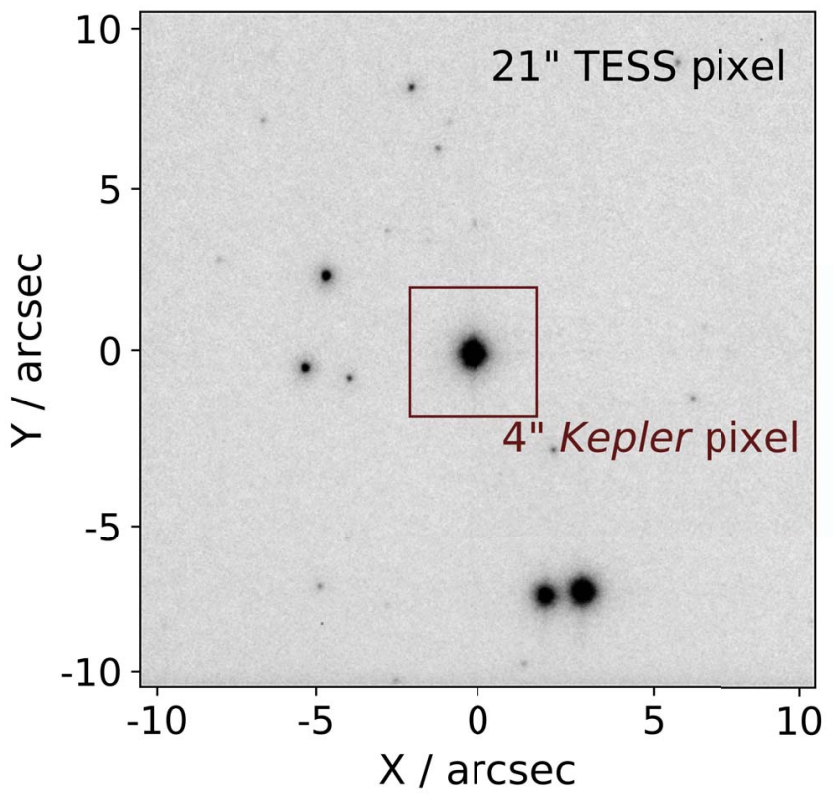

Figure 6. A $21^{\prime \prime}$ square region of sky, the area subtended by a single TESS pixel, from a Robo-AO image centered on a super-Earth-sized planet candidate host, KOI-4725, located at $b=7.6$. For comparison, the pixel size of Kepler has been drawn. The transit signal from this planet candidate, if detected by TESS, would be diluted by multiple additional sources within the same photometric aperture (most TESS fields will, however, be in less crowded fields than the original Kepler field). If not accounted for, the planetary candidate radius would be significantly underestimated due to this contamination; in this illustration, the planet candidate would exhibit a transit depth in uncorrected TESS data similar to an Earth-sized rocky planet. Each of these additional sources in this field is identified in Gaia DR2.

Unlike with the Kepler planet candidates, the dilution from nearby stars detected with high-resolution imaging has already been taken into account in many of the $K 2$ planet candidate's reported radii estimates (e.g., Crossfield et al. 2016). In addition, the literature has significant variations in the planetary radius estimates of many $K 2$ planets, particularly those around late-type stars. This is largely due to highly uncertain stellar parameters derived from photometry alone. Consequently, we do not report radius corrections for the $K 2$ candidates with detected nearby stars in Gaia DR2.

\subsection{Implications for TESS}

TESS, launched in 2018 April, will search nearly the entire sky for transiting planets around bright, nearby stars (Ricker et al. 2014). Simulations estimate that TESS will detect over 10000 exoplanets, including approximately 250 potentially rocky planets (Barclay et al. 2018). With significantly larger pixels than Kepler ( $21^{\prime \prime}$ compared to $\left.4^{\prime \prime}\right)$, the TESS light curves for most targeted stars will have some contamination from nearby stars (see Figure 6). In the case of a transiting planet, this additional flux dilutes the transit signal, resulting in underestimated planetary radii.

Ground-based, wide-field surveys, such as 2MASS or SDSS, typically detect near-equal contrast companions to within separations of 3" (Ziegler et al. 2018a). The recovery of nearby stars to Kepler planet candidates proves that Gaia DR2 is a far more complete census of the stellar population in the vicinity of TESS targets. Gaia specifically is not sensitive to low-contrast, sub-arcsecond companions (although unresolved low-mass 
binaries may be identified, if not characterized, by their presence above the main-sequence using the precise parallaxes and stellar properties resulting from Gaia DR2 (Berger et al. 2018)). Ziegler et al. (2018a) found that for systems with Robo-AO detected nearby stars, the estimated radii of Kepler planet candidates will increase by a factor of 1.54, on average, assuming the planet is equally likely to orbit the primary or secondary star. Using instead only the nearby stars detected by Gaia, including those not detected by Robo-AO, the planet candidates radii estimates will increase by a factor of 2.47 , under the same assumptions. Of course, the stars detected by Gaia DR2 are, in general, much fainter and widely separated and are unlikely to be bound to the primary star (Horch et al. 2014; Ziegler et al. 2018b). The scenario in which the primary and secondary star are equally likely to host the star is not likely and leads to a high occurrence rate of Jupiter-sized planets that has not been observed (Howard et al. 2012). If instead, all planets orbit the primary star, the additional flux from the Gaia detected stars will lead to the radii of planet candidates in multiple systems increasing by a factor of 1.12 , on average.

With Gaia DR2, the properties of a large number of nearby stars not resolved in seeing-limited ground-based surveys will be readily available, greatly improving the initial radius estimates of detected TESS planets. Ultimately, however, the TESS planet candidates will each require ground-based highresolution follow-up observations to identify the close, likely bound stars, as well as provide more precise characterization and confirmation. With Gaia DR2 alone, the radius estimates of 254 Kepler planet candidates would be underestimated due to non-recovery of close binaries which could be detected with high-resolution instruments. Fortunately, the brightness of the TESS targets, typically 2-5 mag brighter than Kepler targets, will allow smaller telescopes with less-costly high-angular resolution instruments, using methods such as speckle (Horch et al. 2014) or lucky imaging (Law et al. 2006), to be able to detect a large fraction of the sub-arcsecond companions which are not recovered by Gaia. In addition, as the TESS targets will be significantly closer than for Kepler, the on-sky angular separation of binaries will increase, allowing a larger fraction of binaries to be detected by diffraction-limited instruments on meter-class telescopes.

In addition, with multiple stars contributing to a single cumulative TESS light curve in which a purported planet transit signal is detected, it may be unclear which star is the source of the brightness dip (i.e., whether the transit is indeed a planet around the bright star, or a faint background eclipsing binary). The Kepler pipeline identifies some astrophysical false positives through a variety of tests, such as significant secondary transit events or in- and out-of-transit centroid shifts (Coughlin et al. 2016). The latter of these tests will be more difficult with the lower resolution and coarser plate scale of TESS.

\section{Conclusion}

We found that the majority of binaries from the Robo-AO Kepler survey with separations greater than $1^{\prime \prime}$ were recovered in Gaia DR2 with magnitude contrasts as large as six. Binaries with separations less than $1^{\prime \prime}$ were typically not recovered, regardless of secondary brightness. We find that the recovery rate of binaries by Gaia is not dependent on position angle. We found 177 nearby stars to Kepler planetary candidates in DR2 that were not detected by Robo-AO. These newly detected stars are faint and likely not bound to the primary, and their impact on the planet candidate radii estimates is likely minimal. Between Robo-AO and Gaia, we found that $18.7 \pm 0.7 \%$ of Kepler planet candidate hosts have nearby stars within $4^{\prime \prime}$. In addition, we found 36 nearby stars around $35 K 2$ planetary candidates, and the $K 2$ planet hosts displayed a significantly lower nearby star fraction rate than the Kepler planet hosts.

With years of observations to come, it is expected that the sensitivity of Gaia will improve in later data releases, converging on the simulated recovery rate reported by Arenou et al. (2017), with most binaries outside of $0 . " 5$ detected. At present, Gaia DR2 will improve initial TESS planet radius estimates by identifying contaminating sources within the same pixel as the planet host star. For precise characterization and confirmation, however, further ground-based high-resolution follow-up observations will be required.

A future analysis will use existing Keck-AO observations of Kepler planet candidates performed by the Robo-AO team, as well as available archival data, to further test the sensitivity to close stellar binaries in Gaia DR2 and subsequent catalogs. The astrometric and photometric precision achieved by Gaia for stars in close proximity will be compared to that of single stars. With the deep imaging available with a large-aperture telescope, we will also be able to confirm or refute the existence of faint, potentially spurious, sources detected by Gaia near bright stars.

This work uses data from research supported by the NASA Exoplanets Research Program, grant \#NNX 15AC91G. C.Z. is supported by a Dunlap Fellowship at the Dunlap Institute for Astronomy \& Astrophysics, funded through an endowment established by the Dunlap family and the University of Toronto. C.B. acknowledges support from the Alfred P. Sloan Foundation. T.M is supported by NASA grant \#NNX 14AE11G under the Kepler Participating Scientist Program. S.M. acknowledges support from the National Science Foundation award AST-1517592. D.H. acknowledges support by the National Science Foundation (AST-1717000) and the National Aeronautics and Space Administration under grant NNX14AB92G issued through the Kepler Participating Scientist Program. The authors thank the Research Corporation for hosting the 2018 Time Domain Astronomy Scialog, where the idea for this project originated.

The Robo-AO team thanks NSF and NOAO for making the Kitt Peak $2.1 \mathrm{~m}$ telescope available. Robo-AO KP is a partnership between the California Institute of Technology, the University of Hawaii, the University of North Carolina at Chapel Hill, the Inter-University Centre for Astronomy and Astrophysics (IUCAA) at Pune, India, and the National Central University, Taiwan. The Murty family feels very happy to have added a small value to this important project. Robo-AO KP is also supported by grants from the John Templeton Foundation and the Mt. Cuba Astronomical Foundation.

This research has made use of the NASA Exoplanet Archive, which is operated by the California Institute of Technology, under contract with the National Aeronautics and Space Administration under the Exoplanet Exploration Program. This work has made use of data from the European Space Agency (ESA) mission Gaia (https://www.cosmos.esa.int/gaia), processed by the Gaia Data Processing and Analysis Consortium (DPAC, https://www. cosmos.esa.int/web/gaia/dpac/consortium). Funding for the DPAC has been provided by national institutions, in particular the institutions participating in the Gaia Multilateral Agreement. 
This work made use of the gaia-kepler.fun crossmatch database created by Megan Bedell.

We thank the anonymous referee for her or his careful analysis and useful comments on the manuscript.

Facilities: PO:1.5m (Robo-AO), KPNO:2.1m (Robo-AO), Gaia.

\section{ORCID iDs}

Carl Ziegler (ii) https://orcid.org/0000-0002-0619-7639

Nicholas M. Law (1) https://orcid.org/0000-0001-9380-6457

Christoph Baranec (ํ) https://orcid.org/0000-0002-1917-9157

Reed Riddle (1) https://orcid.org/0000-0002-0387-370X

Nathan De Lee (1) https://orcid.org/0000-0002-3657-0705

Suvrath Mahadevan (i) https://orcid.org/0000-0001-

9596-7983

Joshua Pepper (1) https://orcid.org/0000-0002-3827-8417

\section{References}

Andrae, R., Fouesneau, M., Creevey, O., et al. 2018, arXiv:1804.09374

Arenou, F., Luri, X., Babusiaux, C., et al. 2017, A\&A, 599, A50

Arenou, F., Luri, X., Babusiaux, C., et al. 2018, arXiv:1804.09375

Bailer-Jones, C. A. L., Rybizki, J., Fouesneau, M., Mantelet, G., \& Andrae, R. 2018, arXiv:1804.10121

Baranec, C., Riddle, R., Law, N. M., et al. 2014, ApJL, 790, L8

Baranec, C., Riddle, R., \& Law, N. M. 2017, arXiv:1709.07103

Baranec, C., Ziegler, C., Law, N. M., et al. 2016, AJ, 152, 18

Barclay, T., Pepper, J., \& Quintana, E. V. 2018, arXiv:1804.05050

Berger, T. A., Huber, D., Gaidos, E., \& van Saders, J. L. 2018, arXiv:1805. 00231

Chabrier, G., Baraffe, I., Allard, F., \& Hauschildt, P. 2000, ApJ, 542, 464
Ciardi, D. R., Beichman, C. A., Horch, E. P., \& Howell, S. B. 2015, ApJ, 805,16

Coughlin, J. L., Mullally, F., Thompson, S. E., et al. 2016, ApJS, 224, 12

Crossfield, I. J. M., Ciardi, D. R., Petigura, E. A., et al. 2016, ApJS, 226, 7

de Bruijne, J. H. J., Allen, M., Azaz, S., et al. 2015, A\&A, 576, A74

Dotter, A., Chaboyer, B., Jevremović, D., et al. 2008, ApJS, 178, 89

Fabricius, C., Bastian, U., Portell, J., et al. 2016, A\&A, 595, A3

Gaia Collaboration, Brown, A. G. A., Vallenari, A., et al. 2018a, arXiv:1804. 09365

Gaia Collaboration, Spoto, F., Tanga, P., et al. 2018b, arXiv:1804.09379

Gaia Collaboration, Prusti, T., de Bruijne, J. H. J., et al. 2016, A\&A, 595, A1

Horch, E. P., Howell, S. B., Everett, M. E., \& Ciardi, D. R. 2014, ApJ, 795, 60

Howard, A. W., Marcy, G. W., Bryson, S. T., et al. 2012, ApJS, 201, 15

Huber, D., Bryson, S. T., Haas, M. R., et al. 2016, ApJS, 224, 2

Janson, M., Hormuth, F., Bergfors, C., et al. 2012, ApJ, 754, 44

Jensen-Clem, R., Duev, D. A., Riddle, R., et al. 2018, AJ, 155, 32

Kraus, A. L., Ireland, M. J., Huber, D., Mann, A. W., \& Dupuy, T. J. 2016, AJ, 152,8

Law, N. M., Hodgkin, S. T., \& Mackay, C. D. 2006, MNRAS, 368, 1917

Law, N. M., Hodgkin, S. T., Mackay, C. D., \& Baldwin, J. E. 2005, AN, 326, 1024

Law, N. M., Morton, T., Baranec, C., et al. 2014, ApJ, 791, 35

Mathur, S., Huber, D., Batalha, N. M., et al. 2017, ApJS, 229, 30

Moe, M., \& Di Stefano, R. 2017, ApJS, 230, 15

Raghavan, D., McAlister, H. A., Henry, T. J., et al. 2010, ApJS, 190, 1

Ricker, G. R., Winn, J. N., Vanderspek, R., et al. 2014, Proc. SPIE, 9143, 914320

The Astropy Collaboration, Price-Whelan, A. M., Sipőcz, B. M., et al. 2018, arXiv:1801.02634

Thompson, S. E., Coughlin, J. L., Hoffman, K., et al. 2018, ApJS, 235, 38

Zhang, Z. H., Pinfield, D. J., Burningham, B., et al. 2013, MNRAS, 434, 1005

Ziegler, C., Law, N. M., Baranec, C., et al. 2018a, AJ, 155, 161

Ziegler, C., Law, N. M., Baranec, C., et al. 2018b, AJ, 156, 83

Ziegler, C., Law, N. M., Baranec, C., Riddle, R. L., \& Fuchs, J. T. 2015, ApJ, 804,30

Ziegler, C., Law, N. M., Morton, T., et al. 2017, AJ, 153, 66 